\section{Tannerella forsythia: patógeno importante en la periodontitis, integrante del complejo rojo}

\begin{abstract}
Tannerella forsythia: important pathogen in periodontitis, member of the red complex
\end{abstract}

\section{Artículo de Revisión}

Donald Ramos-Perfecto ${ }^{1, a}$

1 Universidad Nacional Mayor de San Marcos, Lima,
Perú.

${ }^{a}$ Doctor en Estomatología. Magister en Microbiología.

\title{
Correspondencia:
}

Donald Ramos Perfecto: dramosp@unmsm.edu.pe

Ciudad universitaria UNMSM, Av. German Amezaga 375,

Lima, Perú

ORCID: 0000-0001-5870-3962

\section{Editor:}

Juan Carlos Cuevas-González

Universidad Autónoma de Ciudad Juárez, México.

Conflicto de intereses: el autor declara no tener conflictos de interés.

Fuente de financiamiento: autofinanciado.

Recibido: 21/05/20

Aceptado: 10/06/20

Publicado: 04/08/20

\begin{abstract}
Resumen
Dentro de los microorganismos que forman el complejo rojo, considerado por Socransky, se encuentran algunas bacterias agresivas, como la Tannerella forsythia, esta es un bacilo anaerobio estricto, presente en la enfermedad periodontal, debido a su factor de virulencia, y a su capacidad de subsistir en condiciones óptimas en la bolsa periodontal, esta a su vez estimula la activación de mediadores de la inflamación, que con la cronicidad de la enfermedad daña de manera progresiva el periodonto. Esta revisión resaltara los aspectos fundamentales de la bacteria, así como su participación en la patogénesis de la enfermedad periodontal.
\end{abstract}

Palabras clave: Tannerella forsythia; Factores de virulencia; Diagnóstico; Tratamiento (fuente: DeCS BIREME).

\begin{abstract}
Among the microorganisms that form the red complex, considered by Socransky as aggressive bacteria in periodontitis, such as Tannerella forsythia, a strict anaerobic bacillus, which would be participating with relevance in this pathology, its multiple virulence factors, as well as its capacity to subsist under optimal conditions in the periodontal pocket, such as stimulating the activation of inflammation mediators that with the chronicity of the disease would be progressively damaging the periodontium. In this review, we will learn more regarding this microorganism of great importance in periodontal diseases.
\end{abstract}

Keywords: Tannerella forsythia; Virulence factors; Diagnosis; Treatment (source: MeSH NLM). 


\section{Introducción}

La cavidad bucal es un ecosistema donde tejidos blandos y duros, así como fluidos y microorganismos, coexisten en homeostasis, generando un ambiente de salud al cual llamamos eubiosis. Pero esta armonía puede desnaturalizarse con la aparición de enfermedades como la caries dental y las enfermedades periodontales, es en esta última su presencia se puede dar por múltiples factores, como cierta susceptibilidad del huésped, hábitos nocivos desarrollados, presentes en el paciente y la proliferación de flora polimicrobiana muy virulenta contenida en un biofilm subgingival. Se ha podido determinar que existe una flora bacteriana, (complejo rojo), constituida por Porphyromonas gingivalis (P. gingivalis) ${ }^{1}$, Treponema denticola (T. denticola) ${ }^{2}$ y Tannarella forsythia (T. forsythia $)^{3}$. Estos microorganismos estarían dañando progresivamente las estructuras del periodonto, generando en un inicio lesiones leves en un proceso continuo, donde la respuesta inflamatoria crónica se vuelve dañina, la lesión toma mayor severidad generando un cuadro clínico conocido como periodontitis, donde las estructuras de soporte del diente se van deteriorando, pudiendo generar la pérdida de este ${ }^{4}$.

En relación a las bacterias más relevantes en la periodontitis, T. forsythia es un bacilo con múltiples factores de virulencia que apoya el inicio y progresión de la enfermedad ${ }^{5}$. Este microorganismo, así como los productos generados por su presencia y otros presentes en la bolsa periodontal, pueden diseminarse por vía hematógena pudiendo desarrollar patologías a distancia, así la literatura menciona que estos microorganismos podrían ser un factor de riesgo para la presentación de diversas enfermedades como; aterosclerosis ${ }^{6}$, artritis reumatoide ${ }^{7,8}$, diabetes tipo $2^{9,10}$, cáncer, entre otros ${ }^{11}$. Se ha podido relacionar una mayor presencia de $T$. forsythia en pacientes con índice de masa corporal elevada, pudiendo tener un riesgo en el inicio y progresión de la periodontitis ${ }^{12}$.

El objetivo de la revisión es dar a conocer un microorganismo de suma importancia en las enfermedades periodontales, así como sus factores de virulencia, fisiopatología, diagnóstico, y susceptibilidad a diversos antimicrobianos de utilidad en el apoyo del tratamiento de la periodontitis.

\section{Revisión de la literatura}

\section{Taxonomía}

T. forsythia, fue aislada por primera vez en bolsas periodontales profundas de humanos, a mediados de 1970, siendo considerado por sus características de ser anaerobio y de forma bacilar para pertenecer al grupo de los Bacteroides. Este primer hallazgo lo efectuó Anne Tanner, en el instituto Forsyth ${ }^{13.14}$. Para el 2002 ya con estudios moleculares a base de la secuencia del ARNr 16S del microorganismo, se pudo determinar su separación del género Bacteroides y constituir un nuevo género llamado Tannerella, proponiendo Sakamoto que esta primera especie se llame $T$. forsythensis ${ }^{15}$. Este nombre tuvo un pequeño cambio gramatical para el 2003, de acuerdo al código internacional de nomenclatura de bacterias, llamándose como actualmente la conocemos, T. forsythia ${ }^{16}$.

\section{Morfología y estructura bacteriana}

T. forsythia es un bacilo de aspecto fusiforme, Gram negativo, anaerobio estricto, con un diámetro de 0,3 a 0,5 $\mu \mathrm{m}$, una longitud de 1 a $30 \mu \mathrm{m}$, siendo como promedio de $5 \mu \mathrm{m}$, a nivel central la bacteria presenta un agrandamiento del diámetro de hasta $3 \mu \mathrm{m}$. Es una bacteria inmóvil, que presenta por fuera de su pared celular una cubierta llamada capa $S$ de aproximadamente de $10 \mathrm{~nm}$ de ancho ${ }^{13}$. Su pared celular está constituida por una membrana externa e interna, separados por una zona densa de electrones que tiene aproximadamente $16 \mathrm{~nm}$ de ancho. T, forsythia por parecerse a un bacilo filamentoso, podría confundirse con el género Fusobacterium al ser observado microscópicamente, teniendo que diferenciarse de éste por características como; no producir ácido butírico, no fermentar carbohidratos por lo general, ausencia de crecimiento en caldo glucosado, con actividad tipo tripsina y morfológicamente en el centro de su cuerpo bacteriano presenta un incremento de su volumen $3,5,14$.

\section{Factores de Virulencia}

\section{Capa S}

Viene a ser una cubierta externa de la bacteria, compuesta de proteínas alineadas regularmente. Está relacionado con la adhesión e invasión bacteriana, así también atenúa la respuesta del huésped.

Esta capa $S$ tiene una dimensión aproximada de $10 \mathrm{~nm}$ de ancho por $10 \mathrm{~nm}$ de alto. en su conformación se ha podido identificar dos glicoproteínas de alto peso molecular de 220 y $210 \mathrm{kDa}$, codificados por los genes $t f_{s} A$ y $t f s B$ respectivamente. Se han observado residuos de glucosa en esta capa, el cual promovería una mayor interacción con bacterias como Fusobacterium nucleatum (F. nucleatum), que interactuarían con receptores tipo lectina, lo cual promovería la formación de biopelículas mixtas. También se ha podido identificar que esta capa media la adhesión e invasión a células del carcinoma epitelial de la boca ${ }^{3.5 .17}$.

\section{Proteína BspA}

Es una proteína de superficie cuya siglas BspA, provienen de las palabras en inglés ( Bacteroides, surface, protein, A), esta pertenece a una familia de proteínas ricas en leucina, presenta dos regiones D1 y D2 en la porción $\mathrm{N}$ terminal y en la porción $\mathrm{C}$ terminal contiene 4 dominios de tipo inmunoglobulina.

La BspA se une a los componentes de la matriz extracelular, fibronectina y fibrinógeno, así también induce la producción de citocinas proinflamatorias que participan en la reabsorción ósea, en monocitos mediante la vía dependiente de TLR-2. También estimula la expresión de la quimiocina CXC en células murino de preosteoblastos, las actividades antes mencionadas tienen implicancia en 
la colonización, adhesión, el reclutamiento de neutrófilos, inflamación y destrucción tisular ${ }^{3,5,18}$.

Otros estudios han podido identificar que la proteína BspA tiene un rol importante en el desarrollo del biofilm, ya que estaría participando en la interacción bacteriana, como la coagregación y ya constituido el biofilm, estaría generando un mecanismo de evasión de la respuesta inmune ${ }^{19}$.

\section{Lipoproteína BflP}

Es una lipoproteína de superficie considerada una evasina, que muestra una actividad sobre fibroblastos gingivales, para producir elevados niveles de IL- 6 y TNF- $\alpha$. Esta lipoproteína induce la producción del factor nuclear kappa B por fibroblastos. La IL-6 induce reabsorción ósea por inducción o activación de osteoclastos, mediado por una señal vía Toll-like receptor 2. Así también esta lipoproteína inicia un proceso de apoptosis por activación de la caspasa 8 , de células de tipo leucocitos y linfocitos ${ }^{3,5}$.

\section{Proteasa PrtH}

Codificada con el gen $p r t H$ con un peso molecular de $478 \mathrm{kDa}$, esta enzima hidroliza el péptido sintético N-Benzoil-Val-Gly-Arg-p-Nitroanilida, asi como causa hemolisis a la sangre de caballo. Se ha podido identificar que la PrtH estaría participando como factor de desprendimiento o separación de la célula a un sustrato. Así también esta proteasa aumenta el potencial oxidativo mitocondrial en las células, lo que generaría una producción de IL-8 a partir de las células separadas 5 .

De lo antes mencionado se puede concluir que la $\mathrm{PrtH}$ estaría involucrado en la degradación del epitelio gingival, así como la inducción de la citocina proinflamatoria IL-8, desempeñando un papel que agravaría la patología periodontal ${ }^{3,20}$.

\section{Sialidasas}

Son enzimas que cortan enlaces $\alpha$ cetosodicos entre el ácido siálico y los residuos glucósidos de glicoproteínas del huésped, siendo dos las sialidasas expresadas en el microorganismo SiaHI y NanH. Este tipo de proteína está presente en virus, protozoarios y hongos. Su presencia es sumamente significativa en las lesiones periodontales como en la periodontitis relacionada a generar una modificación en la capacidad de respuesta del huésped ante una infección bacteriana, así también $\mathrm{NanH}$ estaría tomando un rol importante en la colonización bacteriana. Se ha podido identificar que estas enzimas contribuyen a la destrucción del tejido del huésped de forma in vivo, así como estaría relacionado a un crecimiento in vitro de T. forsythia, ya que sería utilizada para obtener $\mathrm{N}$-acetil-ácido-murámico, un factor de crecimiento para esta bacteria ${ }^{3,5,21}$.

\section{Enolasa}

Es una enzima citolítica que puede ser secretada o expuesta en la superficie bacteriana, pudiendo funcionar como receptores plasminogenos. La enolasa de T. forsythia tiene la actividad enzimática de convertir 2-fosfoglicerato a fosfoenolpiruvato y gran capacidad de unión como activación del plasminógeno, lo que generaría la degradación del fibrinógeno. Así también induce la producción de citocinas proinflamatorias como IL-1 $\beta$, IL-6, IL-8 y TNF- $\alpha$ en la línea celular de monocitos THP-1 humano, Esta enzima de T. forsythia estaría potenciando la respuesta inflamatoria en la periodontitis ${ }^{22}$.

\section{Dipeptidil Aminopeptidasa IV}

Es una serina proteasa producida por T. forsythia que degrada gelatina, colágeno tipo I y III. Esta enzima rompe el dipeptido X-Pro o X-Ala u extremo $\mathrm{N}$ terminal de las proteínas. Como es sabido el colágeno tipo I es rico en secuencia de X-Pro y X-Ala, siendo este el primer constituyente de los tejidos del periodonto, así el 80-85\% de colágeno en la gingiva es de tipo I, como el $90 \%$ lo es en el hueso alveolar. El colágeno de tipo III representa el $15 \%$ del colágeno total del periodonto. Esta enzima estaría participando en gran forma en la progresión de la periodontitis ${ }^{23}$.

\section{Mirolisina}

Es una metaloproteasa de la familia pappalysin, como zimógeno se autoactiva proteolíticamente extracelularmente en el enlace Ser 54-Arg 55, secretada por T. forsyhtia, la cual corta múltiples componentes de la vía del complemento generando una forma de protección del patógeno hacia esta vía. Así también degrada el péptido antimicrobiano LL-37, el cual es secretado por células epiteliales y del sistema inmune en lugares o sitios de infección. Esta molécula puede conducir a desarrollar nuevas formas de tratamiento como la elaboración de potentes inhibidores peptidomiméticos de esta proteasa, el cual podría contribuir a empeorar las condiciones de enfermedad ${ }^{24}$.

\section{Molécula Chaperona GroEL}

Es una molécula presente en células procariota cuya función es ayudar al plegamiento de otras proteínas, siendo un homólogo de la proteína 60 de Shock térmico humano, su implicancia en la patología periodontal estaría en una mayor respuesta inflamatoria, ya que estaría induciendo una producción de IL-6 y IL-8 por fibroblastos gingivales y ligamento periodontal. Así también GroEL asociado con la IL-17 estarían produciendo una sinergia incrementándose la producción de prostaglandina E2, promoviendo la inhibición del gen que expresa la osteoprotegerina, generando así un incremento de la reabsorción ósea. La IL-17, es considerada un factor agravante de la inflamación ya que es capaz de estimular a los fibroblastos y células epiteliales en la producción de IL-6 y IL-8 principalmente ${ }^{25}$.

\section{Ácido Nonulosonicos}

Son azúcares similares al ácido siálico, de 9 carbonos, $\alpha$ cetoácidos, presentes en la superficie bacteriana. Estudios recientes sugieren que el ácido nonulosonico podría desempeñar un papel como imitadores moleculares de los ácidos siálicos en células eucariotas, contribuyendo en la capacidad del patógeno para evadir o modular la respuesta inmune. 
La glucosilación de la superficie de T, forsythia por ácido nonulosonicos, juega un papel en la fase inicial de la infección, mediante la supresión de la respuesta inmunitaria proinflamatoria, permitiendo la persistencia de T. forsythia en el huésped, así como de otras bacterias presentes en el surco gingival. Así también se ha demostrado que la capa $S$ glucosilada de algunas cepas de T. forsythia suprimen la producción de mediadores proinflamatorios IL-1B, TNF- $\alpha$ y IL-8 en macrófagos U937 y fibroblastos gingivales humanos, esto en la etapa inicial de la infección ${ }^{26}$.

Otro factor que podría considerarse de virulencia, ya que favorece la formación de biofilm subgingival, la coagregación, estimulación del crecimiento bacteriano, así como su sinergia, se daría por la coexistencia de un consorcio entre T. forsythia y otros microorganismos del complejo rojo como $P$. gingivalis y $T$. denticola. Así también la interacción de T. forsythia con $F$. nucleatum estarían favoreciendo la reabsorción ósea en ratones ${ }^{27-29}$.

\section{Fisiopatología de la Tannerella forsythia}

T. forsythia es una bacteria anaerobia que bien puede habitar el surco gingival a profundidad, es básicamente un microorganismo comensal de la cavidad bucal, su presencia en el biofilm dental subgingival genera un consorcio de subsistencia con otras bacterias del complejo rojo, así como una sinergia entre ellas, para poder proliferar y establecerse en ese medio. En todo proceso infeccioso el primer paso es tener la capacidad de adherirse a la célula huésped, esta seda principalmente por componentes presentes en la capa $S$ y por glicoproteínas como BspA. Por su metabolismo proteolítico tiene que degradar estructuras celulares, compuestas por colágeno y proteínas, es aquí donde participan proteasas como la PrtH, y la serina proteasa Dipeptidil aminopeptidasa IV, que tiene función colagenolítica. Así también la actividad de enzimas como la enolasa y la lipoproteasa BflP, estarían generando daño al tejido gingival y hueso alveolar, mediando la producción de citocinas tipo IL-6, IL- $1 \beta$. IL- 8 y TFN- $\alpha$, que estarían generando una reabsorción ósea progresiva. También este microorganismo presenta un mecanismo de evasión de la respuesta del huésped, ya sea por su capa $S$ o por la activación de la apoptosis por la lipoproteína BflP, que reduciría la presencia de leucocitos y linfocitos.

A medida que el microorganismo se asienta en la profundidad del surco crevicular su capacidad de actuar conjuntamente con microorganismos tipo $P$. gingivalis y T. denticola, generarían un mayor dańo al periodonto. Este proceso al llevarse de forma crónica va a generar un daño progresivo del tejido gingival, ligamento periodontal y hueso alveolar principalmente, produciendo los signos clásicos de la periodontitis como; sangrado, pérdida de inserción, bolsa periodontal, movilidad dental principalmente, características que podrían agravarse generando la pérdida de la pieza dentaria $3,5,20,30$.

\section{Diagnóstico microbiológico}

T. forsythia es una bacilo un poco difícil de cultivar, aunque su condición de ser un microorganismo comensal de la cavidad bucal ha permitido identificarlo en muestras de saliva ${ }^{31}$, fluido gingival ${ }^{32}$ y bolsas periodontales 33 , pero es en este último, donde se ha podido determinar su presencia en mayor proporción, así como se ha identificado sus múltiples características de crecimiento, como desarrollarse en un medio libre de oxígeno donde existe una flora polimicrobiana, en constante consorcio con otros microorganismos para su proliferación.

La forma de recuperarla de surcos profundos de $4 \mathrm{~mm}$ a más, requiere un adecuado aislamiento de este microorganismo, siendo la muestra más común recuperada la de biofilm subgingival, esta se obtiene previo aislamiento relativo de la zona, así como el retiro de la placa supragingival con gasa estéril. Luego se puede colocar un cono de papel de $\mathrm{N}^{\circ} 30$ ó 40 , esperando de 20 a 40 s, para que el cono se cargue con el contenido de la bolsa periodontal. También se pueden utilizar otros instrumentos para la toma de muestra en esta zona, pudiendo ser curetas o sondas periodontales, instrumentos más rígidos que podrían cargarse con el ingreso de estas al surco gingival. Una vez cargado el instrumento este es llevado a un medio de transporte como el BHI (caldo-infusión cerebro corazón), Caldo Brucellla, Tioglicolato o el medio VMGA- III. Estas muestras son llevadas al laboratorio y se realizan una serie de diluciones, utilizando las diluciones de $10^{-3} \mathrm{a}$ $10^{-5}$, tomando de ellas una alícuota de $100 \mu \mathrm{L}$ y sembrando por diseminación en un medio enriquecido como el agar sangre, suplementado con hemina $5 \mu \mathrm{g} /$ $\mathrm{mL}$ y vitamina $\mathrm{K} 1 \mu \mathrm{g} / \mathrm{mL}$. Una vez hecha la siembra los medios son incubados en condiciones de anaerobiosis con $80 \%$ de $\mathrm{N}_{2}, 10 \%$ de $\mathrm{H}_{2}$ y $10 \%$ de $\mathrm{CO}_{2}$, a una temperatura de $37^{\circ} \mathrm{C}$ por un periodo que puede durar de 1 a 2 semanas ${ }^{33,34}$.

Algunas pruebas que se utilizan para complementar el diagnóstico de T. forsythia, serian la observación microscópica reconociendo su morfología bacilar y una reacción positiva a la coloración Gram, dar positivo a la prueba CAAM, y un análisis por medio de un kit API 32 ayuda a la identificación de este microorganismo ${ }^{33,35}$.

El reconocimiento de algunas características bioquímicas también ayuda a identificar a este microorganismo, como: presentar actividad positiva para alfa-glucosidasa, Beta-glucosidasa, sialidasa, y ser indol negativo ${ }^{26}$.

Una prueba enzimática de apoyo al diagnóstico, que identifica una enzima tipo tripsina, producida por el microorganismo, se efectúa con la prueba BANA, el cual permite identificar la degradación del compuesto Benzoil-DL-Arginina-2-Napthylamide. Esta prueba es útil para la identificación de todo el complejo rojo ${ }^{36}$.

Pruebas serológicas como Elisa para identificar inmunoglobulinas producidas por la presencia de T. forsythia se podría utilizar como diagnóstico rápido ${ }^{32}$. Así también algunas pruebas moleculares son utilizadas, como; sondas de ADN ${ }^{37}$, PCR convencional ${ }^{34}$, PCR en tiempo real ${ }^{38}$, y PCR multiplex ${ }^{39}$, son algunas pruebas que pueden ayudar al diagnóstico microbiológico. 


\section{T. forsythia y su relación con la periodontitis}

De las patologías más prevalentes en la cavidad bucal son las enfermedades periodontales las que se pueden presentar en formas leves como severas, siendo una de las formas severas la periodontitis, la que estaría relacionado con la pérdida de la estructura dental, esta patología se presenta en aproximadamente el 10\% de la población mundial ${ }^{40}$. Siendo su origen multifactorial, donde el factor microbiano tiene gran importancia y de este conjunto los microorganismos bacilares, Gram Negativo, anaerobios estrictos, destaca T. forsythia, que acompańada de otros microorganismos virulentos como P. gingivalis, T denticola, Prevotella intermedia, F. nucleatum y otros van dañando el periodonto. En relación a $T$. forsythia, está bien documentado como su capa $S$, una estructura proteica, permite la adhesión a células y la colonización bacteriana. Así también múltiples enzimas como proteasas DPP IV, enolasa, sialidasas y otras que degradan y estimulan una producción de citocinas proinflamatorias, que generarían mayor daño con la cronicidad de la enfermedad. Su capacidad de activar la apoptosis, generar sinergia con las bacterias del complejo rojo hace de esta bacteria muy activa en la periodontitis 3,5 .

\section{Tratamiento antimicrobiano}

Reconociendo que el factor microbiano es muy relevante en la periodontitis, distintos protocolos se han utilizado con diferentes antimicrobianos de amplio espectro para reducir o eliminar la presencia de las bacterias anaerobias estrictas en la bolsa periodontal, entre ellas T. forsythia, entendiendo que esta bacteria no actúa sola sino acompañada con un grupo de microorganismo muy virulentos, formando en conjunto el biofilm subgingival. Por tanto, es necesario reducir el biofilm subgingival a una mínima cantidad, primero con procedimientos mecánicos como lo es el raspaje y alisado radicular, considerado el gold standard del tratamiento para esta patología, esta fase inicial bien ejecutada lograra remover muchos microorganismos entre ellos T. forsythia.

Como complemento a este procedimiento se puede utilizar diferentes antimicrobianos que tienen muy buen accionar sobre T. forsythia y otros que forman el complejo rojo. De estos antimicrobianos destaca por su amplio espectro y fácil dosificación la moxifloxacina, que en dosis de $400 \mathrm{mg} / 24 \mathrm{~h} / 7$ días ${ }^{41,42}$ es de ideal uso en la periodontitis. Otros antibióticos como la amoxicilina a una dosis de $500 \mathrm{mg} / 8 \mathrm{~h} / 7$ días, metronidazol $500 \mathrm{mg} / 8 \mathrm{~h} / 7$ días, ciprofloxacino $500 \mathrm{mg} / 24 \mathrm{~h} / 7$ días, son menos eficaces, pero de igual manera se podría utilizar. Algunas combinaciones como la amoxicilina más metronidazol ${ }^{43.44}$ han demostrado generar una sinergia muy buena que daría un gran apoyo al tratamiento, así también una buena forma de acompańamiento al tratamiento de la periodontitis es el uso de amoxicilina más ácido clavulánico ${ }^{42}$. También la combinación amoxicilina más tinidazol podría utilizarse, ya que estudios recientes han demostrado su actividad contra T. forsythia y otros patógenos importantes, la dosis de tinidazol es de $500 \mathrm{mg} / 12-24 \mathrm{~h} / 7$ días ${ }^{45}$.
Otro antibiótico que se han utilizado con buenos beneficios en el apoyo al tratamiento de la periodontitis, es la azitromicina con una dosis de $500 \mathrm{mg} / 24 \mathrm{~h} / 3$ días, por vía oral ${ }^{46}$.

En el caso de los antimicrobianos locales, la clorhexidina podría ser de uso a una concentración de $0,12 \% /$ $12 \mathrm{~h} / 10$ días, para luego utilizar una concentración de 0,05/12 h/ 2 meses, tiempo en el cual se debería evaluar el beneficio del tratamiento.

Algunos procedimientos que pueden ser utilizados para reducir la presencia de T. forsythia de la bolsa periodontal así como del complejo microbiano presente puede ser; la terapia fotodinámica ${ }^{47}$, el uso de ozono líquido o gas ${ }^{48}$, o el uso del láser Er:YAG ${ }^{49,50}$.

El uso de antimicrobianos y diversos procedimientos a base de agentes químicos y físicos, para reducir la flora microbiana en la periodontitis debe ser siempre acompañado de una muy buena fisioterapia por parte del paciente, reduciendo así el biofilm supragingival a la mínima cantidad, evitando que está a medida que se desarrolle pueda internalizar al surco gingival. Por tanto, se recomienda un mantenimiento del tratamiento efectuado cada 2 ó 3 meses.

\section{Conclusiones}

Tannerella forsythia es un microorganismo comensal de la cavidad bucal, su presencia en los surcos profundos de los pacientes con periodontitis, su capacidad de adherirse a la célula huésped, liberar una variedad de proteasa con capacidad colagenolítica, modular el sistema inmune en su inicio y promover la producción de varias citocinas proinflamatorias en el surco crevicular, que generaría una destrucción progresiva del periodonto. Así también con otras bacterias conforman un consorcio sumamente agresivo para el huésped. Todo lo mencionado de este microorganismo da sustento a su relevancia como patógeno en la periodontitis.

\section{Referencias bibliográficas}

1. Ramos PD, Moromo NH, Martinez CE. Porphyromonas gingivalis predomínate en la periodontitis crónica. Odontol Sanmarquina. 2011;14(1):34-38.

2. Ramos PD, Avila CM, Levano V. Treponema denticola: patógeno en procesos periodontales y pulpares. Odontol Sanmarquina. 2012;15(2):38-41

3. Holt S, Ebersole J. Porphyromonas gingivalis, Treponema denticola and Tannerella forsythia: the "red complex", a prototype polbacterial pathogenic consortium in periodontitis. Periodontol 2000. 2005;38:72-122.

4. Suzuki N, Yoneda M, Hirofuji T. Mixed Red-Complex bacterial infection in periodontitis. Int $\mathrm{J}$ Dent. 2013;2013:I87279. DOI: 10.1155/2013/587279.

5. Sharma A. Virulence mechanisms of Tannerella forsythia. Periodontol 2000. 2010;54:106-116.

6. Schenkein H, Papapanou P, Genco R, Sanz M. Mechanisms underlying the association between periodontitis and atherosclerotic disease. Periodontol 2000. 2020:83:90-106. 
7. Ogrendik M. Rhematoid Arthritis is an autoimmune disease caused by periodontal pathogens. International Int J Gen Med. 2013;6:383-386.

8. Martinez-Rivera J, Xibillé-Friedmann D, Gonzalez-Christian J, De la Garza-Ramos M, Carrillo-Vasquez $S$, Montiel-Hernandez J. salivary ammonia levels and Tannerella forsythia are associated with rheumatoid arthritis: A cross sectional study. Clin Exp Dent Res. 2017;3:107-114.

9. Quintero AJ, Prada P, Inostroza CM, Chaparro A, Sanz AF, Ramirez VL, et al. Presencia de Porphyromonas gingivalis, Tannerella forsythia, Treponema denticola y Aggregatibacter actinomycetemcomitans en el biofilm dental subgingival de pacientes diabéticos tipo 2: estudio transversal. Rev Clin periodoncia implantol Rehabil Oral. 2011;4(2):54-58.

10. Polak D, Sarnui T, Nishimura F, Shapira L. Diabetes as a risk for periodontal disease-plausible mechanisms. Periodontol 2000. 2020;83:46-58.

11. Malinoswki B, Wesierska A, Zalewska K, Sokolowka M, Bursiewicz W, Socha M. et al. The role of Tannerella forsythia and Porphyromonas gingivalis in pathogenesis of esophageal cancer. Infect Agent Cancer. 2019;14:3. DOI: $10.1186 / \mathrm{s} 13027-019-0220-2$.

12. Haffajee A, Socransky S. Relation of body mass index periodontitis and Tannerella forsythia. J Clin Periodontol. 2009;36:89-99.

13. Tanner A, Listgarten M, ebersole L, Strzempko M. Bacteroides forsythus sp. Nov., a Slow-Growing, fusiform Bacteroides sp.from the Human Oral Cavity. Int J Syst Bacteriol. 1986;36(2):213-221.

14. Tanner A, Izard j. Tannerella forsythia, a periodontal pathogen entering the genomic era. Periodontol 2000. 2006; $42: 88-113$.

15. Sakamoto M, Suzuki M, Umeda M, Ishikawa I, Benno Y. Reclassification of bacteroides forsythus(Tanner et al. 1986) as Tannerella forsythensis corrig., gen. nov., comb. nov. Int J Syst Evol Microbiol. 2002;54:841-849.

16. Maiden M, Cohee P, Tanner A. Proposal to conserve the adjectival form of the specific epithet in the reclassification of Bacteroides forsythus Tanner et al. 1986 to the genus Tannerella forsythia corrig., gen. nov., comb. nov. Request for an Opinion. Int J Syst Evol Microbiol. 2003;53:2111-2112.

17. Sekot G, Posh G, Messner P, Matejka M, Rausch-Fan X, Andrukhov O. et al. Potential of the Tannerella forsythia S-layer to Delay the Immune Response. J dent Res. 2011:90(1):109-114.

18. Sharma A, Inagaki S, Honmma K, Sfintescu C, Baker P, Evans R. Tannerella forsythia-induced Alveolar Bone Loss in Mice Involves Leucine-rich-repeat BspA Protein. J Dent Res. 2005;84(5):462-467.

19. Inagaki S, Kuramitsu H, Sharma A. Contact-dependent regulation of a Tannerella forsythia virulence factor, BspA, in biofilms. FEMS Microbiology Letters. 2005;249:291296.

20. Iniesta M, Herrera D, Serrano J, Sanz M. Analisis de los factores de virulencia de los patógenos de asociación fuerte con la periodontitis: Aggregatibacter actinomyce- temcomitans, Porphyromonas gingivalis y Tannerella forsythia. Quintessence. 2008;18(2):109-115.

21. Homna K, Mishima E, Sharma A. Role of Tannerella forsythia NanH sialidase in Epithelial Cell Attachment. Infect Immun. 2011;79(1):393-401.

22. Lee J, Jung Y, Jun H, Choi B. Pathogenic potencial of Tannerella forsythia enolase. Mol Oral Microbiol. 2016:31:189-203.

23. Yost S, Duran-Pinedo A. The contribution of Tannerella forsythia dipeptidyl aminopeptidase IV in the breakdown of collagen. Mol Oral Microbiol. 2018;33(6):407-419.

24. Radisky E. Mirolysin structures open a window on gum disease. IUCrJ. 2020;7:3-4.

25. Jung Y, Choi Y, An S, Lee H, Jun H, Choi B. Tannerella forsythia GroEL indices inflammatory bone resorption and synergizes with interleukin-17. Mol Oral Microbiol. 2017;32:301-313.

26. Bloch S, Tomek M, Friedrich V, Messner P, Schaffer $\mathrm{CH}$. Nonulosonic acids contribute to the pathogenicity of the oral bacterium Tannerella forsythia. Interface Focus. 2019;9(2):20180064. DOI: 10.1098/rsfs.2018.0064.

27. Sharma A, Inagaki S, Sigurdson W, Kuramitsu H. Sinergy between Tannerella forsythia and Fusobacterium nucleatum in biofilm formation. Oral Microbiol Immunol. 2005;20:39-42.

28. Yoneda M, Yoshikane T, Motooka N, Yamada K, Hisama K, Naito T, Okada I. et al. Stimulation of growth of Porphyromonas gingivalis by cell extracts from Tannerella forsythia. J Periodont Res. 2005;40:105-109.

29. Settem R, TaherA, Honma K, Stafford G, Sharma A. Fusobacterium nucleatum and Tannerella forsythia induce synergistic Alveolar Bone Loss in a Mouse periodontitis Model. Infect Immun. 2012;89(7):2436-2443.

30. Tomi N, Fukuyo Y, Arakawa S, Nakajima T. Pro-inflammatory cytokine oroduction from normal human fibroblasts is induced by Tannerella forsythia detaching factor. J Periodont Res 2008;43:136-142.

31. Saygun I, Nizan N, Keskiner I, Bal V, Kubar A, Acikel C, et al. Salivary infectious agents and periodontal disease status. J Periodontol Res. 2011;46:235-239.

32. Onishi H, Arakawa S, Nakajima T, Izumi Y. Levels of specific immunoglobulin $G$ to the forsythia detaching factor Tannerella forsythia in crevicular fluid are related to the periodontal status. J Periodont Res. 2010;45:672680.

33. Van Winkelhof A, Loos B, Van der Reijden W, Van der Veiden U. Porphyromonas gingivalis, Bacteroides forsythus and other putative periodontal pathogens in subjects with and without periodontal destruction. J Clin Periodontol. 2002;29:1023-1028.

34. Narayanan D, Hamlet S, Cullinan M, Davies R, Ellwood $\mathrm{R}$, Bird P, et al. The distribution of Tannerella forsythia in an adolescent and adult population. J Periodont Res. 2005;40:482-488.

35. Mayorga-Fayad I, Lafaurie G, Contreras A, Castillo D, Baron A, Aya M. Microflora subgingival en periodontitis crónica y agresiva en Bogota, Colombia: un acercamiento epidemiológico. Biomedica. 2007;27:21-33. 
36. De Andrade J, Feres M, De Figueiredo L, Salvador S, Cavalca Sh. The ability of the BANA test to detect different levels of $P$. gingivalis, $T$. denticola and $T$. forsythia. Braz Oral Res. 2010;24(2):224-230.

37. Tanner A, Kent Jr R, Kanasi E, Lu SH, Paster B, Sonis S, et al. Clinical characteristics and microbiota of progressing slight chronic periodontitis in adults. J Clin Periodontol. 2007;34:917-930.

38. Abiko Y, Sato T, Mayanagi G, Takahashi N. Profiling of subgingival plaque biofilm microflora from periodontally healthy subjects and from subjects with periodontitis using quantitative real time PCR. J Periodont Res. 2010;45:389-395.

39. Papone V, Verolo C, Zaffaroni L, Batlle A, Capo C, Bueno $\mathrm{L}$, et al. Detección y prevalencia de patógenos periodontales de una población con periodontitis crónica en Uruguay mediante metodología convencional y metagenómica. Odontoestomatología. 2015;17(25):23-32.

40. OMS Salud Bucal. [consultado el 25 de marzo de 2020] accesible en: https://www.who.int/topics/oral_health/es/

41. Ardila C, Bedoya-García J. Antimicrobial resistence of Aggregatibacter actinomycetemcomitans, Porphyromonas gingivalis and Tannerella forsythia in periodontitis patients. JGAR. 2020;S2213-7165(20)30053-9. DOI: https://doi.org/10.1016/j.jgar.2020.02.024.

42. Cruz E, Ramirez J, Contreras A. Moxifloxacino como coadyuvante en el tratamiento de las periodontitis. Rev Clin Periodoncia Implatol Rehabil Oral. 2014;7(3):200-208.

43. Teughels W, Feres M, OUD V, Martin C, Matesanz P, Herrera D. Adjuntive effect of systemic antimicrobial in periodontitis therapy. A systematic review and meta-analysis. J Clin Periodontol. 2020;29. DOI:10.1111/ jcpe. 13264
44. Liaw A, Miller C, Nimmo A. Comparing the periodontal tissue response to non-surgical scaling and root planning alone, adjunctive azithromycin or adjunctive amoxicillin plus metronidazole in generalized chronic moderate-to-severe periodontitis: a preliminary randomized. Controlled trial. Aust Dent J. 2019;64(2):145-152.

45. Rams T, Sautter J, Van Winkelhoff A. Comparative In Vitro Resistane of Human Periodontal Bacterial Pathogens to Tinidazole and Four other Antibiotics. Antibiotics. 2020;9:68. DOI:10.3390/antibiotics9020068.

46. Oliveira A, Costa F, Nogueira L, Cortelli Sh, Oliveira P, Aquino D, et al. Azithromycin and Full-Mouth Scaling For the Treatment of Generalized Stage III and IV Periodontitis: A 6-Month Randomized Comparative Clinical Trial. Braz Dent J. 2019;30(5):429-436.

47. Segarra-Vidal M, Guerra-Ojeda S, Soraya L, LópezRoldan A, Mauricio M, Aldasoro M. et al. Effects of photodynamic therapy in periodontal treatment: A randomized, controlled clinical trial. J Clin Periodontol. 2017;44:915-925.

48. Huth K, Quirling M, Lenzke S, Paschos E, Kamereck $\mathrm{K}$, Brand K, et al. Effectiveness of ozone against periodontal pathogenic microorganisms. Eur J Oral Sci. 2011;119:204-210.

49. Milne T, Coates D, Leichter J, Soo L, Williams S, Seymour G, et al. Periodontopathogen levels following the use of an Er:YAG laser in the treatment of chronic periodontitis. Aust Dent J. 2016;61:35-44.

50. Cobb Ch. Lasers and the treatment of periodontitis: the essence and the noise. Periodontol 2000. 2017;75:205295 
